\title{
Émotions, rationalité, densité temporelle et manifestation de valeurs
}

Pierre Livet

\section{(2) OpenEdition}

Journals

Édition électronique

URL : http://journals.openedition.org/ress/59

DOI : $10.4000 /$ ress. 59

ISSN : 1663-4446

Éditeur

Librairie Droz

Édition imprimée

Date de publication : 1 mai 2009

Pagination : 7-23

ISBN : 978-2-600-01303-1

ISSN : 0048-8046

Référence électronique

Pierre Livet, «Émotions, rationalité, densité temporelle et manifestation de valeurs », Revue européenne des sciences sociales [En ligne], XLVII-144 | 2009, mis en ligne le 01 mai 2012, consulté le 22 avril 2019. URL : http://journals.openedition.org/ress/59 ; DOI : 10.4000/ress.59 
Pierre LIVET

\section{ÉMOTIONS, RATIONALITÉ, DENSITÉ TEMPORELLE ET MANIFESTATION DE VALEURS}

\section{INTRODUCTION}

Les liens entre les notions de rationalité, d'émotion, de valeurs et d'expérience du temps peuvent sembler ténus. Il devient cependant souhaitable de s'interroger sur les relations entre ces concepts si l'on remarque que tous ont quelque rapport avec une sensibilité à des différences, et particulièrement à des différences qui persistent dans leur forme. Inversement, une fois qu'on a adopté cette perspective, on dispose d'une voie d'analyse qui peut modifier nos présupposés, au moins ceux qui portent sur les rapports entre la notion de rationalité et celle d'émotion, et sur notre accès aux valeurs. Davantage, l'adoption de cette perspective «différentialiste » nous évite de devoir supposer des valeurs et une rationalité qui existeraient en soi, puisque penser en termes de différences relativement stables, c'est se borner à utiliser les ressorts les plus élémentaires de nos processus cognitifs, dont les matériaux de base sont justement issus de notre sensibilité à des différences stables (qu'on songe aux frontières entre objets, dans la perception). Les différences peuvent rester stables alors même que les termes qui diffèrent sont mal définis ou sont changeants, et que leur statut d'objet n'est pas évident; ainsi un nuage en mouvement par rapport à d'autres nuages en mouvement reste identifiable tant que nous pouvons l'englober dans une pseudo-frontière perceptive qui assure la connexité de sa forme par rapport aux autres nuages. Cette frontière reste une structure d'un réel complexe qui comprend aussi nos processus d'extraction des frontières perceptives, voire même les processus de stabilisation de ces frontières comme reconnaissables non seulement par nous-mêmes mais par autrui. C'est cette interaction différentielle entre le nuage, les autres nuages, nos processus perceptifs et les processus intersubjectifs de reconnaissance qui reste stable, ce ne sont pas les objets que seraient les nuages et nos processus neuronaux et plus largement corporels, ainsi que ceux des autres individus.

Si cette analogie a quelque portée, et si nous nous intéressons aux structures de ces modes d'accès au monde par celles des différences qui ont une certaine persistance, alors nous pouvons considérer la rationalité comme une gamme ordonnée d'exigences de stabilité: stabilité sous forme de cohérence, et mise en ordre stable entre différents types de cohérence. Notre expérience du temps peut se comprendre - en toute première analyse - comme un rapport entre différentes dynamiques qui fait émerger certaines formes d'ordre, ou, dit autrement, comme des modes de rapports entre les vitesses de ces dynamiques - le temps nous est sensible quand il se densifie ou quand il se dilate. Nous pouvons aussi concevoir les émotions à partir des différences entre nos attentes implicites ou explicites et 
ce qui nous survient, et les distinguer selon leurs dynamiques - les émotions occurrentes intenses ont une traîne irréductible, alors que les sentiments évaluatifs peuvent se remplacer les uns les autres rapidement quand nous envisageons tour à tour plusieurs options. Enfin, c'est par des différences persistantes entre conduites - entre les conduites supposées les plus communes et donc les plus stables dans une communauté, et des conduites qui mettent durablement en exergue certaines différences- que nous pourrons nous assurer que nos semblables peuvent prétendre être dans leurs activités les porteurs de certaines valeurs.

Beaucoup plus modestement, cet article se propose seulement de montrer que si l'on adopte cette perspective différentialiste, on peut jeter quelques lumières et restaurer quelque cohérence dans trois problèmes: celui des distorsions de nos choix préférentiels par rapport aux exigences de la théorie du choix rationnel à la Savage, dans des situations expérimentales de délibération en incertitude (de choix entre différentes loteries); celui d'ébaucher des principes de choix entre les différentes manières de faire se succéder nos actions; celui de faire la différence entre des déclarations d'adhésion à des valeurs - ou des justifications d'une conduite au nom de telle valeur- et des conduites qui peuvent nous donner confiance dans la capacité d'autrui à se comporter en accord avec ces valeurs.

\section{LES «BIAIS» DES CHOIX EXPÉRIMENTAUX}

Partons donc du problème des incohérences de nos choix avec les présupposés de la théorie du choix rationnel de Savage, et plus simplement avec la maximisation de l'espérance d'utilité, tels que nous les présentent les expériences de Kahneman et Tversky ainsi que leur école. Assurément, il s'agit de choix qui ne sont pas forcément ceux de la vie «réelle», entre autres parce qu'on présente au sujet le choix entre des loteries souvent constituées par des nombres qui désignent des gains ou pertes ainsi que des probabilités. Il est peu probable que nous évaluions les situations d'incertitudes en termes de probabilité, et que nous sachions toujours quel «gain» nous allons retirer de telle situation. Ces biais ou incohérences sont cependant encore plus flagrants si l'on utilise des présentations en termes de différences qualitatives. Et quoi qu'il en soit, notre approche différentialiste doit les prendre au sérieux. En effet, ils constituent des différences stables puisque répétables d'un groupe de sujet à un autre - quelque sens que les sujets donnent aux données présentées- si bien qu'ils font partie de ces structures d'interaction avec le monde dont nous avons parlé plus haut.

Rappelons les plus saillantes de ces incohérences ${ }^{1}$.

1) Nous préférons un gain certain mais inférieur à la combinaison d'un gain de probabilité bien plus faible mais supérieur, d'un gain égal mais de probabilité inférieure et d'un gain nul très peu probable.

2) Entre un gain légèrement supérieur mais de probabilité très légèrement inférieure et un gain légèrement inférieur mais de probabilité très légèrement

1 Telles qu'elles ressortent par exemple des expériences relatées dans Choice, Values and Frames, Kahneman and Tversky eds., Cambridge University Press, 2000. 
supérieure, en revanche, nous préférons le premier, qui est supérieur en espérance d'utilité. Ces deux choix sont incohérents entre eux, du point de vue d'une rationalité guidée par l'espérance d'utilité (ils constituent le paradoxe d'Allais).

3) Nous préférons (en majorité) les gains non pas certains mais proches de la certitude, même s'ils sont inférieurs à des gains plus élevés mais plus incertains, et cela même si les espérances d'utilité sont les mêmes. Nous n'avons donc pas simplement une préférence pour la certitude pure, du genre «un tien vaut mieux que deux tu l'auras », et nous sommes incohérents avec 2), puisque l'égalité entre les espérances d'utilité ne nous rend pas indifférents.

4) Nous préférons les pertes plus grandes mais plus incertaines à des pertes plus faibles mais certaines - notre préférence pour la certitude est ici inversée, mais si cela peut compter formellement comme incohérence avec 1) et 3), ce renversement est aisément explicable.

5) Nous préférons un gain très élevé mais très risqué à un gain très faible mais certain de même espérance d'utilité (par exemple, 5000 euros à 1/1000 contre 5 euros certains, ce qui a fait penser à une surestimation des petites probabilités), ce qui est incohérent avec 1) et 3), et aussi avec 2), puisqu'ici les espérances d'utilité sont les mêmes.

6) Nous ne traitons pas de manière symétrique les gains et les pertes. Ainsi, lorsqu'on nous présente deux programmes médicaux, le premier, A, étant censé sauver avec certitude 200 personnes d'une épidémie qui en aurait fait mourir 600 , le second, B étant censé sauver les 600, mais avec seulement une probabilité d'un tiers, nous préférons le programme certain. Quand maintenant on nous présente A comme laissant mourir avec certitude 400 personnes sur 600 , et B comme nous donnant seulement deux chances sur trois de laisser mourir 600 personnes, nous préférons le programme B. C'est d'abord en contradiction avec 2 (les espérances d'utilité sont égales), puis c'est incohérent avec 1 et 3 . Il semble que ces incohérences qui sont elles-mêmes incohérentes entre elles ne peuvent pas se laisser reformuler d'une manière cohérente (la rationalité en théorie de la décision, pourtant, n'exige pas que nous n'ayons pas, par exemple, de préférence pour ce qui est plus certain, elle exige seulement que nous conservions cette préférence de manière cohérente). Nous allons voir que ce n'est pas le cas, si nous recourons dans nos choix à des formes d'évaluation qui ne prennent un sens intuitif que si nous les comprenons comme des émotions complexes.

\section{LA CONCEPTION DIFFÉRENTIALISTE DES ÉMOTIONS}

Il nous faut donc esquisser la conception «différentialiste» des émotions que nous avons évoquée. L'idée générale, soutenue dans Emotions et rationalité morale $^{2}$, est qu'une émotion est la perception et la résonance affective d'une diffé-

2 Paru aux PUF, 2002. 
rence entre la dynamique des attentes implicites propres à notre activité - ou notre passivité - du moment, et la dynamique impliquée par de nouveaux traits de la situation, quand nous appréhendons ces différences selon nos désirs et préférences. Une différence entre deux dynamiques peut se nommer un différentiel. Une objection à cette idée est que les émotions peuvent être passives et statiques. Or d'une part nous ne sommes jamais totalement passifs, mais toujours disposés envers notre environnement selon une certaine orientation, d'autre part le repos est lui-même une dynamique, celle de l'inertie. L'objection peut être reprise: il est des situations que nous apprécions de manière affective alors qu'elles ne présentent pas de différentiel consciemment perceptible, parce qu'elles sont usuelles. On peut l'admettre à la limite, et l'on pourrait nommer «appréciations affectives » les états ainsi déclenchés, mais ils doivent être en cohérence avec les émotions occurrentes et différentielles que nous ressentirions si nous passions d'une situation indifférente à cette situation appréciée, même si elle est usuelle. On peut aussi les considérer comme des émotions réduites à l'état de dispositions émotionnelles. Enfin l'objection peut être poursuivie: si nous nous attendons à un plaisir - par exemple celui ressenti en entendant pour la nième fois un passage favori d'une pièce musicale- nous devrions pas éprouver d'émotion, selon cette théorie. Ce n'est pas exact. D'une part, nous attendre à un plaisir est déjà anticiper un différentiel entre la situation présent où nous n'éprouvons pas encore de plaisir et celle où nous l'éprouverons. D'autre part, entre l'anticipation et la perception, il reste toujours un différentiel, la seconde étant bien plus riche que la première. Nous ressentirons donc bien l'émotion liée à ces différentiels.

Cependant, pour les problèmes qui nous occupent, nous avons affaire à des états affectifs un peu particuliers. Il s'agit de ce que nous pouvons éprouver quand nous envisageons une branche d'une loterie, avec son gain et la probabilité de ce gain, que nous envisageons ensuite une branche d'une autre loterie, et que nous comparons les deux. Eprouvons nous vraiment des émotions en constatant la différence entre le gain espéré et notre statut présent (si on le suppose à 0 ), ou en constatant la différence entre la certitude de notre état présent, et l'incertitude, l'improbabilité du gain futur? On peut penser qu'il ne s'agit que d'évaluations qui se font sans résonance affective bien évidente. Assurément, il ne s'agit pas d'émotions fortes, puisque d'une part nous n'avons pas encore fait notre choix, ni gagné ou perdu le gain envisagé, et qu'il ne peut donc s'agir d'émotions occurrentes produites par l'irruption de cette nouvelle situation. Cela d'ailleurs nous permet d'envisager la branche d'une loterie, puis une branche d'une autre loterie, sans que soit sensible la «traîne» qu'aurait dû déclencher une première émotion produite par exemple par l'espérance d'un gain, ou la crainte d'une perte. Notre délibération exige que nous puissions passer d'une évaluation, voire d'une émotion à l'autre, sans que l'ordre dans lequel nous le faisons ait un effet trop sensible. Or si il s'agissait d'émotions occurrentes, leurs dynamiques impliqueraient de tels effets, que nous analyserons d'ailleurs quand il s'agira de faire se succéder différentes émotions.

Cependant, s'il s'agissait seulement d'évaluations conceptuelles et de calculs, on ne voit pas bien pourquoi nous différerions de ce que nous prescrit la théorie du choix rationnel. Plus précisément, la combinaison particulière d'espérances d'utilité que nous proposerons pour rendre cohérentes les incohérences relevées n'aurait pas de privilège sur d'autres combinaisons, si nous ne lui donnions pas la 
signification d'émotions plus intégratives que les autres. Il s'agit en effet de privilégier ce que nous appellerons d'une part le soulagement exultant, d'autre part la fierté de la prudence (ou la fierté prudentielle). Quand nous avons choisi une loterie qui nous offre un gain supérieur à celui d'une autre loterie, mais au prix d'un risque plus grand, nous pensons pouvoir éprouver, quand nous aurons la chance d'obtenir ce gain, un supplément de satisfaction qui tiendra à ce que la certitude du gain désormais acquise dépassera ce que nous pouvions espérer, qui était seulement une espérance de gain, donc un gain pondéré par l'anxiété de sa faible probabilité. Bien évidemment, l'espérance d'utilité ne peut pas produire ce supplément de satisfaction, par définition. Et cela parce que par définition aussi, elle ne porte que sur des évaluations de résultats qui ne peuvent pas différer de l'attente qu'elle suscite. Autrement dit, l'espérance d'utilité est en elle-même une neutralisation des effets émotionnels, si nous les pensons comme liés à des différentiels. Puisque ce que nous nommons soulagement exultant tient à un tel effet différentiel (la différence entre la certitude du gain et sa simple espérance) il satisfait à tout le moins des conditions nécessaires, selon nous, pour rentrer dans la catégorie des émotions. Le mode affectif opposé, avons-nous dit, est celui de la fierté prudentielle. Là encore, il s'agit de trouver une satisfaction supplémentaire à l'espérance d'utilité propre à un gain qui, tout en étant inférieur à celui d'une autre loterie, est assuré avec une probabilité supérieure. Nous intensifions en quelque sorte la satisfaction procurée par chaque unité de notre gain en fonction de la supériorité de probabilité qui lui est attachée. Là aussi, il s'agit d'un effet qui n'est pas produit par la seule espérance d'utilité. Il ne tient cependant pas, comme le précédent, à ce que la réalisation du gain dépasse sa simple attente. Il tient plutôt à ce que, ayant d'avance renoncé au gain supérieur en préférant la sécurité, nous pensons éviter ou atténuer tout regret, parce que même si nous n'obtenons pas notre gain inférieur, nous aurons cependant toujours une justification pour l'avoir fait. Autrement dit, si il y a déception de notre attente - ce qui est un différentiel nous pourrons au moins partiellement l'annuler. La fierté de la prudence ne se justifie donc que si elle oppose en quelque sorte d'avance un contre-feu à l'émotion pénible que pourra créer un différentiel ultérieur - non plus, cette fois, celui créé par la réalisation du gain au lieu de sa simple espérance, mais au contraire, le différentiel créé par l'absence de réalisation de nos espérances. Le soulagement exultant s'explique donc par une sensibilité au possible différentiel positif créé par le gain futur, l'espérance d'utilité est supposée neutre émotionnellement, et la fierté prudentielle est une sorte de contre-sensibilité à un possible différentiel négatif créé par la déception de nos espérances. Il s'agit donc bien, selon nos critères, d'émotions, et elles jouent des rôles symétriques par rapport à l'espérance d'utilité, ce qui explique qu'il suffise de tenir compte de ces deux émotions pour expliquer nos choix. Nous pourrons d'ailleurs montrer, en analysant les différentes modalités de nos expériences du temps, que ces émotions qui pourraient paraître sophistiquées et peu immédiates correspondent en fait à des sortes d'échanges entre modalités temporelles (par exemple échanger une densification négative du temps contre une densification positive, ce que réalise le soulagement exultant) et qu'elles sont donc fondamentales.

Ce ne sont cependant pas des émotions occurrentes ordinaires, puisque nous les ressentons par avance, avant même que les loteries soient tirées, et que les gains ou l'absence de gain soit effective. Pouvons nous ressentir des émotions en 
anticipant une situation? Il semble que pour être ému, il faille nous placer soit en réalité, soit par une imagination vivace, dans la situation même. Or ni le soulagement exultant ni la fierté prudentielle ne tiennent simplement à ce que nous nous plaçons par l'imagination dans telle ou telle situation. Elles tiennent aussi à ce que tout en imaginant la possibilité de telle situation sous tel aspect, nous nous plaçons dans une autre situation et perspective: tout en imaginant la possibilité de réalisation du gain supérieur, nous sommes en train de considérer le risque supérieur pris; tout en imaginant la possibilité d'absence du gain inférieur, nous sommes en train de considérer la supériorité de notre choix en termes de sécurité, et à chaque fois, l'attitude dans laquelle nous nous plaçons pour considérer soit le gain supérieur soit l'absence du gain inférieur est une attitude comparative, qui considère un mode de la différence avec l'autre loterie (ici une différence de gain) sous un autre aspect par lequel nous voyons les choses (dans les deux cas, les qualités de probabilité des branches des deux loteries). Nous éprouvons donc bien un différentiel, comme dans toute émotion, mais ce différentiel, nous l'éprouvons à partir d'un point de vue qui diffère de lui et en termes d'aspects (nous visons le gain à partir de la probabilité) et en termes de référent (nous le visons à partir d'une comparaison avec la probabilité de l'autre loterie). On pourrait dire qu'il s'agit d'émotions au cube, si on classait les émotions en fonction du nombre de différentiels, de décalages nécessaires pour les éprouver. Cela implique aussi qu'il ne s'agit pas d'émotions occurrentes usuelles, puisque celles-ci vont nous orienter durablement dans un seul sens, par leur traîne. Ici au contraire, nous devons soit ne pas être trop sensibles aux effets de traîne, pour pouvoir combiner différents aspects et points de vue, soit savoir combiner de manière similaire d'une évaluation à l'autre ces effets de traîne. Une telle capacité de combinaison exige un système affectif évolué, et pénétré de comparaisons et d'évaluations cognitives déjà sophistiquées. Il s'agit donc d'états affectifs, mais qui ont certainement exigé un apprentissage, ce qui implique l'acquisition de dispositions émotionnelles. Nous avons donc des chances d'être avec eux plus proches des «appréciations affectives » ou des sentiments, pris comme appréciations et dispositions émotionnelles cohérentes avec des émotions occurrentes, que des émotions occurrentes usuelles, du genre de la peur ou de la colère. Ce qui nous importe, c'est qu'il s'agit d'états certes évaluatifs, mais qui ne peuvent pas être neutres affectivement et émotionnellement, comme l'est en principe l'évaluation par les seules espérances d'utilité.

Pour pouvoir montrer que ces deux émotions, le soulagement exultant et la fierté prudentielle, rendent cohérents les «biais» que nous avons énumérés, il nous faut cependant leur donner une représentation formelle, et pour ce faire, nous allons aller au plus simple et utiliser le cadre formel de l'espérance d'utilité. C'est là un choix pragmatique, pour pouvoir mieux convaincre le lecteur attaché à la théorie classique du choix rationnel. Nous n'avons pas d'hypothèse sur la manière effective dont nous, humains, procédons formellement pour ces évaluations, ni même un modèle des processus d'évaluation que les appréciations affectives dont nous parlons devrait suivre. Notre représentation par l'espérance d'utilité se veut simplement sur le mode du «comme si», et son but est seulement de montrer qu'on peut retrouver une cohérence dans nos choix apparemment si divergents. 


\section{REPRÉSENTATION DES DEUX ÉMOTIONS PERTINENTES}

Comment donner une représentation du soulagement exultant? L'idée est de partir du gain supérieur supposé acquis et donc certain. Ce qui nous fait exulter, c'est donc ce qui dans cette expérience du gain acquis, dépasse ce que nous pouvions espérer. Mais comme nous comparons deux branches de deux loteries, nous devons aussi tenir compte du contraste avec ce que l'autre loterie nous donnait comme espérances. Nous partons de l'obtention certaine du gain supérieur (Gsup x 1) et en défalquons les espérances que nous avons de ce gain supérieur, ainsi que les espérances propres au gain inférieur, en soustrayant les recouvrements entre les deux.

Comme nos loteries sont indépendantes et ont chacune deux branches, un gain positif et un gain nul, cela nous donne 4 situations possibles. Dans S1, avec la probabilité (p1xp2), la loterie L1 nous donne le gain supérieur, et la loterie L2 nous donne le gain inférieur. Dans S2, avec la probabilité (p1(1-p2), L1 donne le gain supérieur et L2 0. Dans S3, avec la probabilité (p2(1-p1)), L1 nous donne 0 et L2 le gain inférieur. Dans S4, avec la probabilité (1-p1) (1-p2), L1 et L2 nous donnent 0 . Une fois les recouvrements entre gains éliminés (ainsi dans la situation Gsup (p1x p2) nous devons défalquer ce qui tient à $\operatorname{Ginf}(\mathrm{p} 1 \mathrm{x}$ p2)), et nous arrivons alors à la formule ${ }^{3}$ :

$$
\mathrm{E}=\left(\mathrm{Gsup}^{1}-\mathrm{G} \inf ^{2}\right) \times \mathrm{p} 2(1-\mathrm{p} 1)+\left(\mathrm{G} \sup ^{1}-0\right)(1-\mathrm{p} 1)(1-\mathrm{p} 2) .
$$

Nous représentons donc finalement l'exultation par la différence qui selon les situations est soit entre les deux gains, soit entre le gain supérieur et le gain nul, pour tenir compte des deux possibilités des loteries. Et comme ce à quoi nous sommes sensibles dans ce surplus, c'est à ce qui $n$ 'est pas attendu dans cette supériorité de gain, nous associons finalement à ce surplus de gain non pas la probabilité des situations dans lesquelles nous obtiendrions ce gain supérieur, mais au contraire de celles dans lesquelles nous n'attendons pas un tel gain, à savoir S3 et S4.

Mais il ne s'agit que de ce qui, dans le soulagement exultant, justifie l'exultation. Il nous faut aussi estimer l'ampleur du soulagement. Or nous estimons cela, rappelons-le, avant de réellement savoir si nous obtiendrons le gain supérieur. Comme nous envisageons de prendre un risque plus élevé, nous ressentons une anxiété additionnelle (additionnelle, parce que propre au surplus de l'exultation et parce que liée à une prise de risque supérieur à celle de l'autre loterie). Il nous faut donc représenter aussi cette anxiété additionnelle. Pour ce faire, nous partons cette fois non de l'hypothèse de l'obtention du gain supérieur, mais de son manque, ce qui revient à prendre le gain supérieur comme quantité négative. Il faut cependant

3 Nous pouvons vérifier le bien fondé de cette formule en représentant l'ensemble des situations possibles par un cube, qui a pour axe x les gains, pour y les probabilités de 0 à 1 assignées à L1, pour $\mathrm{z}$ les probabilités de 0 à 1 assignées à L2. Gsup x $1 \mathrm{x} 1$ est représenté par la totalité du cube, et de ce cube nous avons à retrancher les différents volumes qui correspondent aux espérances des différents gains. Une telle représentation nous permet d'identifier aisément les recouvrements qu'il faut retrancher, et, dans le cas de l'anxiété additionnelle, quels volumes représentent les gains «manquants attendus». 
en défalquer, comme précédemment, ce qui pouvait être attendu, certes non pas comme gains, mais aussi comme manques à gagner - là encore, en évitant les recouvrements: - $\left(\operatorname{Gsup}^{1} \mathrm{x} 1\right)$, le gain se transformant en gain manqué, moins les manques «attendus » (plutôt qu' «espérés »). Ils sont de $\mathrm{G} \sup ^{1}$ (1-p1) et de G inf ${ }^{2}$ (1-p2). Là encore il faut défalquer le recouvrement: $\operatorname{Ginf}^{2}(1-\mathrm{p} 1)(1-\mathrm{p} 2)$. Il reste: $\mathrm{AA}^{+}=-\left[\mathrm{G} \sup ^{1} \mathrm{x}\right.$ p1- $\mathrm{G}$ inf $\left.{ }^{2}(\mathrm{p} 1(1-\mathrm{p} 2))\right]$, avec un signe négatif devant l'expression entre crochets, puisqu'il s'agit d'anxiété. Or cela revient à partir de l'espérance d'utilité du gain supérieur, qui cumule dans p1 les probabilités des situations S1 et $\mathrm{S} 2$, et à en défalquer le gain inférieur avec la probabilité de la situation S3 (S4 est intervenue, mais dans le recouvrement). Le simple calcul a conduit alors à un effet de chiasme: pour l'exultation, qui concerne l'obtention du gain supérieur, nous considérions finalement les probabilités des situations S3 et S4, dans lesquelles nous n'attendions pas le gain supérieur; pour l'anxiété additionnelle, qui concerne le manque du gain supérieur, nous considérons finalement des probabilités des situations $\mathrm{S} 1$ et $\mathrm{S} 2$, dans lesquelles nous attendons le gain supérieur. A chaque fois, ce sont les probabilités des situations contraposées qui sont dominantes. Le soulagement exultant - ou l'anxiété résiduelle, si l'anxiété additionnelle domineest finalement donné par la combinaison de $\mathrm{E}$ et de $\mathrm{AA}+$.

La représentation de la fierté prudentielle est plus directe. Elle tient à une comparaison entre les situations $\mathrm{S} 2$, de probabilité p1(1-p2) dans laquelle nous obtenons le gain supérieur, mais pas l'inférieur, et $\mathrm{S} 3$, de probabilité p2(1-p1), dans laquelle nous obtenons le gain inférieur, mais pas le supérieur. Comme nous nous satisfaisons du Gain inférieur et que nous nous appuyons sur sa supériorité de probabilité, nous avons:

$\mathrm{FP}=\operatorname{Ginf}^{2} \mathrm{x}((\mathrm{p} 2(1-\mathrm{p} 1)-\mathrm{p} 1(1-\mathrm{p} 2))$, ce qui, comme $\mathrm{p} 2(1-\mathrm{p} 1)=\mathrm{p} 2-\mathrm{p} 1 \mathrm{x}$ p2, et $\mathrm{p} 1(1-\mathrm{p} 2)=\mathrm{p} 1-\mathrm{p} 1 \mathrm{x}$ p2 se réduit à FP $=$ Ginf2 $(\mathrm{p} 2-\mathrm{p} 1)$.

Jusqu'ici nous n'avons raisonné que sur des gains. Il nous faut maintenant considérer les pertes. Kahneman et Tvserky ont montré qu'il n'y avait pas une parfaite symétrie entre nos choix concernant des gains et nos choix concernant des pertes. Cela a une explication émotionnelle. Dans le domaine des gains, nous sommes attirés par ce que le hasard peut nous offrir comme gain, alors que dans le domaine des pertes, nous sommes attentifs à ce que nous pouvons contrôler, dont nous sommes par là responsables. Or un ersatz de contrôle nous est donné par ce à quoi nous pouvons nous attendre - avec l'idée quelque peu stoïcienne que nous sommes responsables de nos décisions en fonction de ce que nous savions pouvoir attendre, mais que nous ne sommes pas responsables de ce qui vient du hasard. Nous devons donc redéfinir nos deux émotions. Nous ne parlerons plus de soulagement «exultant», mais simplement de soulagement, puisqu'il va maintenant reposer non pas sur l'obtention d'un gain, mais sur le fait que le hasard nous aurait permis d'éviter une perte (nous supposons toujours pour simplifier que chaque loterie comporte deux branches, l'une donnant une perte, l'autre 0).

Nous raisonnons de manière similaire cependant. Pour la base du soulagement, nous allons aussi considérer l'évitement complet de la perte Psup, et nous défalquerons de cet évitement complet ce qui pourrait être attendu comme évitement de perte, en évitant les recouvrements. La base de notre soulagement est d'abord de $\operatorname{Psup}^{1} \mathrm{x}$ p1, renvoyant aux situations S1 et S2. Mais dans la situation $\mathrm{S} 1$, nous avions aussi une perte de $\mathrm{Pinf}^{2} \mathrm{x}$ p1x $\mathrm{p} 2$. Si nous comptons nos pertes 
évitées attendues, nous devons donc la défalquer. Notre base de soulagement BS effective est donc:

BS ${ }^{-}$Psup ${ }^{1} \mathrm{x}$ p1 - $\operatorname{Pinf}^{2} \mathrm{x}\left(\mathrm{p} 1 \mathrm{x}\right.$ p2). On notera une similitude partielle avec $\mathrm{AA}^{+}$. Il y a eu croisement entre l'anxiété additionnelle et le soulagement quand on passe des gains aux pertes, et l'on pressent que cela va expliquer un renversement de préférences.

Pour savoir quel est notre soulagement final, il faut savoir aussi quelle est l'anxiété additionnelle liée à cette perte plus risquée. Cette anxiété tient à la crainte de ne pouvoir éviter la perte, donc porte, dans ce type de loterie, sur la probabilité de la perte nulle, conçue comme un «manque» de la perte supérieure. Nous partons évidemment de $\operatorname{Psup}^{1} \mathrm{x}$ (1-p1). Mais puisqu'il s'agit d'une anxiété additionnelle, il nous faut défalquer l'autre perte, correspondant à la situation S3, qui est Pinf ${ }^{2}$ x p2(1-p1). Notre anxiété additionnelle sera donc de:

$\mathrm{AA}^{-}=-\left[\operatorname{Psup}^{1}(1-\mathrm{p} 1)-\operatorname{Pinf}^{2} \mathrm{x}\right.$ p2(1-p1)]. On notera une similitude partielle avec E, si nous réécrivons les deux expressions: $\mathrm{AA}^{-}=(1-\mathrm{p} 1)\left(\mathrm{Psup}^{1}-\mathrm{Pinf}^{2} \mathrm{x}\right.$ p2) et $\mathrm{E}:(1-\mathrm{p} 1)\left((\right.$ Gsup-Ginf $\left.) \times \mathrm{p} 2+\left(\mathrm{Gsup}^{1}-0\right)(1-\mathrm{p} 2)\right)$.

Dans le domaine des pertes, nous ne pourrions éprouver de fierté prudentielle si malgré une prise de risque légèrement inférieure, notre perte était cependant suffisamment élevée pour que l'espérance d'utilité de notre choix soit supérieure négativement à l'espérance d'utilité du choix opposé. Nous n'éprouverons donc de fierté prudentielle positive que dans la mesure où l'espérance d'utilité de la perte choisie sera inférieure en négatif à celle de l'autre choix.

$\mathrm{FP}^{-}=-\left(\operatorname{Psup}^{1} \mathrm{x}\right.$ p1- Pinf $^{2} \mathrm{x}$ p2).

\section{APPLICATION AUX EXEMPLES EXPÉRIMENTAUX}

Montrons maintenant que ces émotions ou du moins leurs représentations formelles rendent cohérents nos choix expérimentaux.

Le paradoxe d'Allais comporte une loterie à trois branches et non deux, par exemple : la loterie L1 nous propose : 2500 à $\mathrm{p} 1=0,33,2400$ à $\mathrm{p} 2=0,66$ et 0 à $\mathrm{p} 3$ $=0,01$ contre la loterie L2: 2400 à $\mathrm{p}=1$. La loterie L2 nous assure la certitude de gagner 2400 euros (Gcertain).

Nous avons 3 situations de probabilités 0 , celles où nous n'aurions pas Gcertain, mais un autre des gains incertains (donc on aurait soit 2500, soit 2400 soit 0 , et jamais Gcertain). Il reste alors 3 situations possibles : S1 : avoir 2500 (et donc par inclusion 2400) et pas 0 , et Gcertain, de probabilité (p1x 1); S2 : ne pas avoir 2500 , ni 0 , mais 2400 ; de probabilité p2 x 1 ; n'avoir ni 2500 , ni 2400 mais 0 , et Gcertain, de probabilité p3 x 1.

L'exultation correspond à la différence entre $\operatorname{Gsup}_{1}{ }_{1}$ et Gcertain, (puisqu'il n’y a pas de différence possible entre $\operatorname{Gsup}^{1}{ }_{1}$ et un 0 du côté du gain certain), 
pondérée par la probabilité de la situation où l'on n'attendait pas $\operatorname{Gsup}^{1}{ }_{1}$, mais 0 et Gcertain, donc de probabilité p3.

Cela donne $2500 \times 0,01=+25$

L'anxiété additionnelle suppose qu'on a raté $\operatorname{Gsup}_{1}{ }_{1}$ au lieu de l'avoir. On ne peut en défalquer le ratage non attendu de Gcertain, puisqu'on ne peut pas le rater. Elle est pondérée par la probabilité des situations où l'on n'attendait pas de le rater, donc où l'on attendait de l'avoir (de probabilité p1). Elle est de -2500 x 0,33 $=-825$. Il n'y a donc pas de soulagement exultant, mais une anxiété résiduelle de -800 .

La fierté prudentielle est ici de 2400 x (1- les probabilités cumulées de 2500 et de 2400) $=0,01$, donc +24 pour le Gain certain.

Au total, le Gain certain gagne par +799 .

Nous choisissons donc sans conteste le gain certain.

2) Il faut considérer l'autre partie du paradoxe d'Allais:

L3: 2500 x $0,33,0$ sinon.

L4: $2400 \times 0,34$.

$\mathrm{E}=100(0.34 \times 0,67)=22,78+2500(0,66 \times 0,67)=1105,5$, total 1128,33

$\left.\mathrm{AA}^{+}=-(2500 \times 0,33)=825\right)+(2400(0,33 \times 0,66)=-522,72$

total 302,28

$\mathrm{PP}=2400 \times 0,34-0,33=24$

Nous préférons L3, loterie pourtant un peu plus risquée, contrairement à notre préférence précédente pour la certitude.

3) La préférence pour la proximité à la certitude

Prenons la loterie L5: 6000 x 0,45, sinon 0 et la loterie L6: 3000 x 0,9. Les espérances d'utilité sont égales.

$\mathrm{E}=3000(0,9 \times 0,55)=1485+6000(0,1 \times 0,55)=330$, total 1815

$\mathrm{AA}^{+}=6000(0,45)-3000 \times(0,45 \times 0)=2700$

$\mathrm{FP}=3000 \times 0,45=1350$, la balance penche pour L6 et sa proximité avec la certitude.

Autre exemple: 4000 à 0,8 et 0 sinon, 3000 à 1 .

$\mathrm{BSE}=1000(1 \times 0,2)=200+3000(0,2 \times 0)=200$

$\mathrm{AA}=4000 \times 0,8=3200-3000(0,8 \times 0)=3200$

$\mathrm{FP}=3000 \times 0,2=600$,

donc en faveur du gain certain.

4) L'inversion de cette préférence dans les pertes.

Prenons la loterie L7: -4000 x 0,8, 0 sinon, et L8: -3000 certains.

$\mathrm{S}^{-}=4000 \times 0,8-3000 \times(0,8 \times 1)=1200$

$\mathrm{AA}^{-}=-(4000 \times(0,2)-(3000(1 \times 0,2)=-200$

$\mathrm{FP}^{-}=4000 \times 0,8-3000 \times 1=200$, c'est donc en faveur de L7. 
5) La soi-disant surestimation des petites probabilités.

Prenons la loterie L9: $6000 \times 0$, 001 et 0 sinon, contre la loterie L10: $3000 \times 0,002$ et 0 sinon.

$\mathrm{E}=3000(0,002 \times 0,999)=5,994+3000(0,999 \times 0,998)=2991,006$ total 2997.

$\mathrm{AA}^{+}=(6000 \times 0,001=6)-(3000 \times(0,001 \times 0,998)=2,994)$ total 8,994

$\mathrm{FP}^{+}=3000 \times 0,001=3$

C'est donc L9 et ses 6000 qui l'emportent. Ce résultat n'a rien à voir avec une surestimation des petites probabilités, mais tout à voir avec le soulagement exultant.

Nous pourrions aussi prendre comme exemple les loteries que nous avons déjà mentionnées, L11: 5000 à 0,001 et L12: 5 certain,. Là aussi, il ne s'agit pas de surestimer la probabilité $1 / 1000$ par rapport à la probabilité 1 , mais de tenir compte du soulagement exultant en faveur de L11.

E: $4995 \times 1 \times 0,999+5000 \times 0,999 \times 0=4990$

$\mathrm{AA}^{+}: 5000 \times 0,001-5 \times 0,001 \times 0=5$

$\mathrm{FP}^{+}=5$ x $0,999=4,995$. Les 5000 l'emportent.

6) Le biais de présentation positive ou négative des deux programmes

Nous avons déjà mentionné ces deux programmes: on annonce que 600 personnes vont mourir d'une épidémie si on ne fait rien, un programme $\mathrm{A}$ en sauvera certainement 200, un programme B a 1/3 de chances d'en sauver 600 . Quand on présente les choses ainsi, les sujets préfèrent le programme A, ce qu'on attribue à un effet de certitude. Quand on les présente en disant que le programme A en laissera certainement mourir 400 et que le programme B a $2 / 3$ de chances d'en laisser mourir 600 , les sujets préfèrent le programme B (inversion de la préférence pour la certitude dans les pertes).

En fait, la présentation positive nous oriente seulement vers le quadrant positif (les gains).

E: $400 \times 1 \times 2 / 3+600 \times(1 / 3 \times 0)=400 \times 2 / 3$

$\mathrm{AA}^{+}: 600 \times 1 / 3-200 \times 1 / 3 \times 0=600 \times 1 / 3$

BSE $>$ AA par $200 \times 1 / 3$

$\mathrm{FP}^{+}: 200 \times 2 / 3$

Le programme certain l'emporte par $200 \times 1 / 3$

Si on se focalise sur le quadrant négatif, celui des pertes, les choses changent. La fierté prudentielle supposée en faveur de A pour ce qui concerne les gains est maintenant nulle pour les pertes: l'espérance d'utilité de A égale celle de B. Le facteur déterminant est le soulagement en faveur de B.

$\mathrm{BS}^{-}: 600 \times 2 / 3-400 \times 2 / 3=200 \times 2 / 3$

AA: $600 \times 1 / 3-400 \times 1 / 3=200 \times 1 / 3$

Le programme incertain l'emporte par 200x $1 / 3$, ce qui est égal à l'avantage inverse que possède le programme certain dans le domaine positif.

Si l'on tient compte à la fois des deux formes d'émotions comparatives, dans les gains et dans les pertes, le programme A et le programme B sont équivalents. Si l'on oriente la présentation vers les émotions comparatives du domaine des gains, c'est le programme A qui domine, et si on oriente vers les émotions comparatives du domaine des pertes, c'est le programme B qui domine, dans la même proportion. Les biais de présentation se ramènent donc à des focalisations soit sur les émotions comparatives du domaine positif, soit sur celles du domaine négatif. 
Nous avons donc rendu compte de ces apparentes incohérences, qui deviennent cohérentes si nos choix se font essentiellement en fonction des deux émotions complexes que sont le soulagement (exultant pour les gains, simple pour les pertes) et la fierté prudentielle.

Notons que les émotions internes à une loterie, que nous avons écartées ${ }^{4}$, peuvent rendre compte d'autres effets à première vue étranges, comme celui qui va suivre.

La survalorisation du pari avec une petite perte et les émotions de comparaison internes

Slovic a présenté une expérience intéressante. A un groupe, on propose un pari qui donne 7/36 chances de gagner 9 dollars, et 0 sinon. A un autre, on propose un pari similaire, 9 euros à $7: 36$, mais avec 29 chances sur 36 de perdre 0,05 dollar. On demande à chaque groupe d'assigner un rang au pari qui lui est présenté, dans une échelle qualitative qui indique des degrés d'attractivité. Le pari qui comporte la petite perte est rangé plus haut que celui sans perte. Bien sûr, si on propose au même groupe de comparer les deux paris, il va donner un rang inférieur au pari avec une petite perte. Il y a donc un biais qui tient à la présentation isolée. Or celleci ne nous permet plus de disposer d'émotions de comparaison externe entre deux loteries. Nous sommes donc obligés de nous rabattre sur les émotions de comparaison interne. Et le pari avec une petite perte nous propose deux types de soulagement: le soulagement exultant qui tient au gain au lieu de 0 , et le soulagement qui tient à l'évitement de la petite perte. La différence de qualité des deux soulagements nous rend plus sensible à l'exultation par contraste avec le simple soulagement. Dans le cas du pari sans perte, nous n'avons pas cette différence de qualité et la faible attractivité des 9 dollars ne met en pas en saillance l'exultation. Ce qui joue en cette affaire, c'est donc encore une émotion comparative, mais une émotion comparative interne qu'on pourrait dire de troisième ordre, celle qui compare le soulagement exultant du gain et le simple soulagement de la perte.

\section{AFFECTS DIFFÉRENTIELS ET TEMPORALITÉ}

Jusqu'à présent, nous avons utilisé l'approche différentialiste des émotions et des affects pour rendre compte de préférences qui se manifestent lors de ces comparaisons délibératives entre des options qui sont elles mêmes statiques. Mais nous pouvons avoir à faire des choix entre deux manières d'agir qui ne diffèrent pas par leurs gains et probabilités, mais seulement par l'ordre de leur succession. Ainsi, quand nous avons à rendre visite, dans la même ville, à des amis très chers et à un oncle grincheux, nous préférons visiter d'abord l'oncle grincheux, puis nous consacrer aux amis, au lieu de faire les choses dans l'ordre inverse. Or cela semble aller à l'encontre d'une autre préférence, celle du discount temporel, qui nous amène à choisir de jouir plus tôt d'un plaisir moindre au lieu d'attendre plus

4 Si nous adoptons l'approche différentielle, nous exultons d'autant plus d'un gain que l'autre branche de la même loterie était plus probable, et donc présentait une grande différence de probabilité. Mais 
longtemps pour un plaisir supérieur, ce qui revient à donner un poids plus grand à ce dont l'occurrence est plus proche du temps présent. Il semble qu'ici se combinent les émotions de la délibération - qui nous permettent de passer d'un point de vue à un autre sans que l'ordre des points de vue compte beaucoup- et les propriétés des émotions occurrentes, qui présentent des effets de traîne difficilement réductibles. Il faut ajouter que l'anticipation d'événements désagréables produit déjà par elle-même des émotions présentes désagréables qui ont un tel effet de traîne. L'anticipation d'événements agréables produit aussi des émotions agréables, mais leur effet de traîne est diminué par l'anxiété s'il y a incertitude ou par l'étirement du temps de l'attente. Visiter nos amis en premier, c'est risquer de voir la joyeuse atmosphère polluée par l'anticipation négative de la visite à l'oncle grincheux. Visiter nos amis en dernier, c'est pouvoir profiter de l'effet de traîne positif du plaisir de leur rencontre. Or l'effet de traîne de la visite désagréable à l'oncle grincheux semble pouvoir au contraire être inhibé rapidement si nous pouvons passer directement à l'activité agréable du temps passé avec nos amis, alors que si nous le visitons en dernier, la traîne de l'émotion désagréable issue de cette visite ne trouvera pas d'inhibiteur.

Quelles sont les relations entre ces évaluations affectives qui portent sur des ordres différents de succession et nos deux émotions de soulagement exultant et de fierté prudentielle? En fait, la fierté prudentielle est nécessaire pour que le scénario des deux visites puisse se construire, car sinon, nous n'irions tout simplement pas visiter notre oncle grincheux. Nous sommes capables de nous sentir fiers d'avoir tout de même visité notre oncle, une fois que nous aurons accompli cette corvée, et cela au lieu de céder immédiatement à l'attraction de la visite à nos amis. C'est de même une motivation analogue à celle fournie par l'anticipation propre à la fierté prudentielle qui peut nous permettre de lutter contre le discount temporel du futur lointain par rapport au futur proche. On peut alors penser que dans notre délibération entre les deux ordres de visite, nous donnons pour contenu au soulagement exultant la capacité d'un événement joyeux à inhiber un effet de traîne négatif, et à déclencher un effet de traîne positif (le soulagement tient à l'inhibition du négatif, l'exultation à la traîne positive); le contenu de la fierté prudentielle devient celui d'avoir résisté à un plaisir qui profitait en plus de l'effet de surestimation du futur proche, pour ne pas prendre le risque de polluer ce plaisir proche par une anticipation négative.

Plus profondément, les différentiels émotionnels sont indissociables, comme l'avait pensé Varela, de notre expérience du temps. On a montré depuis que quand on nous présentait des visages présentant les expressions de la peur ou de la colère, le temps subjectivement estimé à les regarder était jugé avoir duré plus longtemps et être passé plus vite, que lorsque l'on présentait un visage d'expression neutre - le temps est alors estimé avoir duré moins longtemps et être passé moins vite. Nous pouvons donc opposer une expérience de densification du temps et une expérience de dilatation du temps. Cela s'explique si nous pensons ces notions dans les termes de l'accumulation des repères ${ }^{6}$ qui dans un intervalle de

5 Cf Sylvie Droit-Volet et Warren Meck, «How emotions colour our perception of time», Trends in cognitive sciences, 11 (12) p. 504-515, dec 2007.

6 Ces repères sont les traits saillants pour notre perception, orientés par nos motivations du moment. 
temps donné nous permettent d'estimer la durée. Plus nous sommes sensibles à des repères dans un intervalle donné, plus le temps se densifie, moins les repères sont nombreux pour le même intervalle d'horloge, plus le temps se dilate. Le discount temporel tient alors simplement à ce que nous avons du mal à imaginer une grande richesse de repères temporels pertinents dans le futur lointain, alors que dans le futur proche, cela nous est plus facile. Les incertitudes sur le futur lointain sont ainsi redoublées d'une difficulté à penser que les choses seront encore de la même facture qu'au présent, et présenteront donc la même richesse de repères.

Nos affects «temporels » tiennent, selon l'approche différentialiste, à un changement de densité des repères signifiants pour notre activité, et elles tiennent aussi à la position de ces densités dans le temps vécu. On peut faire les hypothèses suivantes: si la densité se situe dans le présent, nous ne percevons tout simplement pas le passage du temps. Si elle se situe dans le futur immédiat, le temps passe du dilaté au densifié, si elle se situe dans le passé immédiat, le temps entre ce passé et le présent est en train de devenir moins dense. Une densité qui serait dans un avenir plus lointain mais qui serait supérieure à celle des moments précédents permettrait en revanche de combattre la préférence pour le futur proche. Une émotion qui monte implique une densification du temps et la traîne de l'émotion est lié à une re-dilatation du temps. Toute dynamique émotionnelle semble donc supposer une transition de la densification à la dilatation ou l'inverse, voire d'une densification positive à une densification négative ou l'inverse. Un temps dilaté présent orienté vers un temps densifié futur nous apparaît comme encore plus étiré (c'est le temps de l'attente). Quand les repères d'une densité future sont liés à des attentes positives, nous préférons le temps densifié au temps étiré de l'attente et au temps dilaté, et c'est l'inverse quand il s'agit d'attentes négatives. Si nous pensons que nous aurions pu dans l'intervalle de temps considéré, vivre un temps densifié (positivement), la dilatation ou l'étirement nous sont encore plus pénibles, ce que nous pourrions nommer un effet de résonance.

Nous pouvons reprendre sous cet angle l'analyse de la délibération qui nous conduit à déterminer l'ordre des deux visites. Si nous adoptions l'ordre: amis puis oncle grincheux, nous aurions d'abord une densification positive peu avant et pendant la première visite, avec une retombée dans la dilatation ensuite. Cette densification positive elle-même serait peu à peu contrée par un étirement négatif lié à l'attente de la deuxième visite, qui donnerait lieu ensuite à une densification négative, suivie de sa traîne, laquelle comporterait comme seul élément positif le soulagement d'en avoir fini. Il y aurait donc peu de soulagement exultant (sauf si la présence des amis était incertaine, mais cela n'est pas supposé), et aucune fierté prudentielle: nous n'aurions procédé à aucune inhibition de nos attraits émotionnels immédiats, mis à part le fait que nous nous sentons obligés de visiter notre oncle (ce qui peut s'expliquer par des rétorsions familiales possibles). Si nous adoptons l'ordre: oncle grincheux puis amis, nous aurons d'abord une densification négative avant et pendant la première visite, mais elle sera allégée d'une part par l'attente réconfortante de la visite suivante, et par la fierté prudentielle d'avoir inhibé notre attrait immédiat pour cette visite. La traîne négative de cette visite pénible sera inhibée rapidement par la montée en densification liée à une attente rapide - qui ne laisse pas le temps s'étirer- puis à la visite chez les amis, donnant lieu cette fois à un soulagement exultant, non pas pour cause d'incertitude, mais 
pour cause d'échappée à la traîne négative. Et la traîne de l'émotion de la visite restera positive même si nous pourrons regretter que ce soit terminé. Enfin, une fois que nous aurons envisagé les deux scénarios, nous ne pourrons plus envisager leurs rapports sans regretter pendant l'entre deux entre les visites, quand nous avions choisi de voir d'abord nos amis, le contraste entre étirement négatif, lié au regret que les réjouissances doivent laisser place aux ennuis, et l'inhibition de cette traîne négative propre à l'ordre inverse des visites, puis, après la seconde visite, le contraste entre la dilatation négative de son arrière goût amer et la dilatation positive du souvenir des amis, le contraste pendant les deux visites étant aussi en faveur de la visite en premier à notre oncle, puisque nous aurons dans ce cas pollué la joie entre amis que nous aurons pu goûter pleinement dans l'autre cas. On voit que commencer par la visite pénible se trouve finalement conjuguer les effets du soulagement exultant et de la fierté prudentielle, et que les comparaisons inter-périodes sont presque toujours en faveur de cet ordre de visites, à l'exception de la période qui précède tout juste la visite des amis, quand nous l'avions programmée en premier.

Si les affects impliquent que nos temporalités prennent certains aspects, nous sommes bien inversement capables de convertir les temporalités en affects, mais aussi de comparer dans nos délibérations plusieurs manières de vivre le temps. Il est enfin possible de comprendre la fierté prudentielle et le soulagement exultant dans les termes des densification ou dilatation du temps, puisqu'elles sont liées à des dynamiques affectives spécifiques. Prendre des risques plus élevés qu'il n'est normal, c'est supporter plus d'anxiété. L'anxiété nous amène à ré-évoquer sans cesse les possibilités d'échec du projet engagé, et elle combine ainsi densification négative et étirement - puisque pendant les instants où nous ne pensons plus à notre projet, nous devenons moins sensibles aux événements qui ne le concernent pas. Le soulagement exultant de la réussite transforme cette densification négative en densification positive. Si nous n'avions pas été angoissés, nous ne ressentirions pas une telle exultation. Cet affect est donc la transmutation d'une densification négative en la densification positive correspondante. Dans la fierté prudentielle, nous procédons au contraire à la re-dilatation d'une densification possible (résistant ainsi à l'attrait d'un plaisir proche) pour pouvoir diminuer notre anxiété, ce qui revient là aussi, d'après ce que nous venons de dire, à re-dilater la densification négative liée à l'anxiété. Ainsi, dans la fierté prudentielle, nous échangeons une re-dilatation d'une densification positive contre la re-dilatation d'une densification négative, et dans le soulagement exultant, nous échangeons une densification négative contre une densification positive. Les deux émotions s'organisent donc bien de manière symétrique comme nous le pressentions, et leur dualité est liée à la combinaison de deux dualités fondamentales: la bipolarité des affects et la dualité entre densification et dilatation du temps.

\section{MANIFESTATIONS D'ATTACHEMENT À DES VALEURS}

Nos deux émotions du soulagement exultant et de la fierté prudentielle, quand elles sont partagées, peuvent nous conforter dans l'idée que nous partageons les mêmes valeurs. La fierté prudentielle nous permet d'inhiber l'attrait pour la 
satisfaction immédiate de certains désirs et intérêts, pour nous rendre une autre satisfaction plus assurée, et il est donc aisé de voir un tel comportement comme une manifestation de la valeur supérieure que nous donnons à cette autre satisfaction. L'attrait du soulagement exultant peut nous amener à nous engager dans des coopérations qui présentent des risques. En cas de réussite, l'exultation est alors partagée, et cela semble un fondement possible de la constitution de nouveaux collectifs. C'est alors la coopération elle-même qui peut apparaître comme valeur - même si la plupart du temps, la coopération se réalise aussi au nom d'autres valeurs. Si nous relions ce problème de la manifestation et reconnaissance de l'attachement à des valeurs et celui des processus de reconnaissance sociale - au sens de reconnaissance de statuts sociaux aussi bien que de valeurs sociale des actes et des personnes- nous voyons là encore que le soulagement exultant et la fierté prudentielle sont chacun en résonance avec des modes de reconnaissance différents. Le soulagement exultant, nous l'avons dit, est un des moteurs des risques pris par chacun dans l'engagement dans une coopération collective, qui peut amener à la création de nouvelles institutions. La fierté prudentielle est plus «conservatrice». Elle ne permet pas la création de nouvelles institutions, mais elle assure davantage le maintien des institutions et de leurs contraintes face à l'attraction de profits plus immédiats. Le type de reconnaissance lié au soulagement exultant coopératif est plus fusionnel, le type de reconnaissance lié à la fierté prudentielle se situe à l'intérieur d'une institution et de ses différences de statut, les membres de même statut se reconnaissant entre eux, les différences de statut ayant tendance à se conserver.

Malgré le lien de cette question avec les processus de reconnaissance sociale, savoir ce qu'est une valeur en elle-même reste la plupart du temps quelque peu énigmatique. En revanche, que certaines prises de risques en commun, ou inversement la résistance à des possibilités attractives dans le souci d'assurer le long terme, soient liées à des comportements reconnaissables par nos congénères, ce sont là des signes de valorisation peu discutables, et il reste seulement à donner des noms à ces valeurs. Les termes de coopération ou d'entre-aide, ou encore de prudence et de tempérance sont ceux qui viennent immédiatement à l'esprit, mais coopération et tempérance peuvent se mettre au service de diverses causes. A l'inverse, si nous pouvions ramener un comportement à une maximisation en termes d'espérances d'utilités, ce serait là pour nous un signe de ce qu'y sont seulement en jeu des «intérêts». Là encore, nous aurions des difficultés à dire ce que sont en soi nos intérêts. Nous savons simplement que les orientations qu'ils peuvent nous donner pour nos choix sont différentes de celles que peuvent nous donner les différentes versions du soulagement exultant et de la fierté prudentielle qui deviennent, quand ils sont mis en commun et reconnus par d'autres, des signes de sensibilités axiologiques. Par ailleurs, dans le domaine des affects temporels, la ligne de base à laquelle nous identifions les «intérêts » n'est pas exactement celle de la rationalité du choix rationnel. Le discount du futur lointain par rapport au futur proche est le lot commun, et nos raisonnement «intéressés» sont censés obéir à ce discount. La rationalité du choix rationnel, quand elle se veut moins sensible au discount, ne serait-ce que pour éviter les incohérences temporelles (choisir a plutôt que $b$ quand $a$ et $b$ sont dans un futur lointain, mais b plutôt que a quand on se rapprochera de ce futur), semble donc elle-même requérir un comportement qui relève de la fierté prudentielle et qui manifeste une valorisation. 
A vrai dire, on pourrait aussi considérer comme une manifestation d'attachement à des normativités l'obéissance des calculs d'espérance d'utilité à une axiomatique précise, contrainte que n'implique pas la simple recherche locale de la maximisation de l'espérance d'utilité. Au cœur même de la rationalité du choix, nous pourrions donc distinguer ce qui est recherche de valeurs, et position normative, de ce qui est censé décrire les calculs de base liés aux intérêts les plus répandus, et qui correspondrait à un calcul local d'espérance d'utilité avec discount temporel. Mais en fait, nous avons montré que nos choix effectifs répondent davantage aux deux affects que sont le soulagement exultant et la fierté prudentielle qu'à la simple maximisation de l'espérance d'utilité. Le rôle de ces deux affects semble alors ambigu. Sont-ce des processus affectifs de base qui devraient alors être comptés dans nos motivations intéressées, ou bien sont-ce les dispositifs nécessaires pour la manifestation de l'attachement à des valeurs?

En fait, ils sont probablement les deux. D'une part, ils semblent essentiels pour comprendre les motivations de nos choix, qu'elles soient intéressées ou pas - la distinction entre intérêts et valeurs est ici relative, puisque l'on peut dénommer intérêts toute motivation qui est supposée commune à la très grande majorité des individus et dont il suffit donc de reconnaître la fréquence sans en faire une norme. D'autre part, ils auraient pu, dans l'évolution de l'humanité, avoir été réutilisés, quand ils ont été conjoints à des processus de coopération, de mise en commun, de reconnaissance mutuelle, de manière à rendre plus saillantes les manifestations de valeur. Et de notre point de vue différentialiste, c'est surtout par cette réutilisation que les valeurs se différencient, dans une communauté et une culture donnée, de ce que l'on peut considérer comme des intérêts de base; les intérêts, eux, sont bien communément reconnaissables, mais ils n'assurent pas les liens de coopération et de mise en commun.

Nous espérons avoir montré la capacité d'une approche différentialiste des émotions et plus généralement des affects à rendre compte de phénomènes multiples - les «biais» des choix expérimentaux, les effets de discount et de préférence pour certaines successions temporelles, les modes de manifestation des valeurs, elles-mêmes conçues dans une relation de différence avec les intérêts, la différenciation des deux étant plus stable et plus saisissable que l'essence propre de chacun des deux termes. Au cours d'un tel plaidoyer en faveur de cette approche, nous avons pu montrer des liens profonds entre les émotions ou appréciations affectives et l'expérience du temps, dans la dualité de cette expérience entre densification et dilatation, et dans la reprise de ces dualités temporelles et affectives dans les modes de constitution de collectifs et d'institutions. Il n'est pas étonnant qu'en partant d'une perspective différentialiste, nous arrivions à découvrir des dualités, mais la capacité de ces différentes dualités à se relier entre elles est plutôt une heureuse surprise et l'indice que nous disposons là d'instruments d'analyse qui sont riches de potentialités.

\section{Université de Provence}

